\title{
Case-control study of disease determinants for non-typhoidal Salmonella infections among Michigan children
}

\author{
Muhammad Younus 1,2,7, Melinda J Wilkins 1,3,4, Herbert D Davies 1,5, Mohammad H Rahbar1,6, Julie Funk3, \\ Chau Nguyen 1,2, Azfar-E A Siddiqi' ${ }^{1}$, Seongbeom Cho² and Mahdi Saeed*1,2,3
}

\begin{abstract}
Background: Infections with Salmonella serotypes continue to be a significant global public health problem. In addition to contaminated foods, several other sources contribute to infections with Salmonella serotypes. We have assessed the role of socioeconomic factors, exposure to food, and environmental sources in the etiology of nontyphoidal Salmonella infections in Michigan children.

Findings: A case-control study among Michigan children aged $\leq 10$ years was conducted. A total of 123 cases of children with laboratory-confirmed Salmonella infections and 139 control children, who had not experienced symptoms of gastrointestinal illness during the month prior to the interviews, were enrolled. The cases and controls were matched on age-category ( $<1$ year, 2-<6 years and 6-10 years). Data on socioeconomic status, food intake, and environmental exposures, were collected on the queried case and control subjects. After adjusting for race and household-income the final regression multivariable model revealed that Salmonella infections were significantly associated with attendance of a daycare center (adjusted matched odds ratio $=5.00,95 \% \mathrm{Cl}: 1.51-16.58$ ), contact with cats $(\mathrm{MOR}=2.53,95 \% \mathrm{Cl}: 1.14-5.88)$, and contact with reptiles $(\mathrm{MOR}=7.90,95 \% \mathrm{Cl}: 1.52-41.01)$, during the 3 days prior to the onset of child's illness.

Conclusions: Study results suggest that exposure to environmental sources may play an important role in sporadic infections with Salmonella serotypes in children. Additional efforts are needed to educate parents and caretakers about the risk of Salmonella transmission to children from these sources.
\end{abstract}

\section{Findings}

Epidemiologic investigations, including reports from the CDC's FoodNet, have demonstrated higher incidences of Salmonella infections in children compared to adults $[1,2]$. In 2003, the incidences of laboratory-confirmed cases of Salmonella infections reported at FoodNet sites were 122.7 cases per 100,000 population for children aged $<1$ year, 50.6 cases per 100,000 population for children aged 1-4 years, and 10.8 cases per 100,000 population for those aged $\geq 5$ years [3]. During 2002-2006, Michigan Department of Community Health $(\mathrm{MDCH})$, reported 63.96 cases of salmonellosis per 100,000 population for children aged $<1$ year, 12.62 cases per 100,000 population

* Correspondence: saeeda@msu.edu

1 Department of Epidemiology, Michigan State University, East Lansing, Michigan 48824, USA

Full list of author information is available at the end of the article for children aged 1-9 years, and 8.45 cases per 100,000 population for aged $\geq 10$ years [4]. One of the reasons of higher incidence of salmonellosis cited in the literature is the increased case detection of Salmonella infections in children compared to the adult population. In young children with gastrointestinal symptoms, increased detection is likely due to: 1) the parents will seek medical attention and 2) the healthcare provider will more likely submit a sample for culture [5].

The majority of sporadic cases of salmonellosis in the adult population results from consumption of contaminated food and traveling to areas where Salmonella is more prevalent [6]. However, risk factors for salmonellosis in children have not been extensively evaluated in population-based epidemiologic studies. The majority of the studies in children have been conducted in response 
to salmonellosis outbreaks, particularly in daycare centers and nurseries. The risk factors identified in outbreaks may not be similar to those of sporadic cases of salmonellosis since exposures in sporadic cases vary widely. A few studies have identified eating undercooked eggs [7], infant formula [1], attending a day care with a child with diarrhea [1], household member with a gastrointestinal (GI) symptoms [7], and contaminated home environment [8] as risk factors for Salmonella infections in children. It has been suggested that contaminated environmental sources contribute more than contaminated food vehicles in the acquisition of Salmonella infections in children [7]. This report describes a population-based case-control study conducted to identify potential risk factors for sporadic, non-typhoidal Salmonella infections in Michigan children aged $\leq 10$ years.

\section{Methods}

\section{Study design and subjects}

A case-control study was conducted between December 15, 2006 and October 15, 2007 among Michigan children aged $\leq 10$ years. Salmonellosis is a notifiable disease under the Michigan Communicable Disease Rules [9]. Therefore, physicians and laboratories across Michigan are required to report cases of salmonellosis to their local health department (LHD) in either the jurisdiction where the individual with suspected salmonellosis resides or where the reporting facility is located (passive surveillance system). In addition to reporting to the $\mathrm{MDCH}$, LHDs and clinical laboratories send isolates to the $\mathrm{MDCH}$ Bureau of Laboratories for serotyping and the results are entered into the state wide Michigan Disease Surveillance Systems (MDSS).

In this study, cases were defined as children aged $\leq 10$ years with laboratory-confirmed Salmonella infections with permanent Michigan address. Case children were excluded from the study if the case 1) was reported as part of a salmonellosis outbreak investigated by Michigan Department of Community Health (MDCH) officials and 2) had a reported congenital malformation (e.g., birth defect), serious medical condition, or a concomitant infection.

Controls were children aged $\leq 10$ years who were not diagnosed with any enteric infections (e.g., salmonellosis, campylobacteriosis) by a healthcare provider and did not experience any enteric disease symptoms (e.g., diarrhea, vomiting, nausea) during the 30 days prior to the interview day. Controls were excluded if the child had a congenital malformation or serious medical condition. Controls were enrolled by two methods: 1 ) case parent(s) were asked to identify a child of similar age to their own child and 2) using the on-line telephone directory http:// www.whitepages.com. This directory has a reverse address function that allowed the compilation of lists of potential control household phone numbers. Households were called, and after explaining the study's objective, we asked if there were any children aged $\leq 10$ years living in the household. Consented parents or caretakers of these children were interviewed.

\section{Questionnaire and data collection}

Data was collected using a structured questionnaire on sociodemographic characteristics (e.g., age and sex, household income, parental education), child feeding practices (e.g., breast feeding, formula milk, use of pacifier), child rearing (e.g., daycare, pre-school, or elementary school attendance), food exposures (e.g., consumption of milk, meat, poultry, eggs, produce, water) and various environmental exposures (e.g., contact with animals, contact with a person having GI symptoms). The questionnaire is presented under additional file 1.

Exposures were assessed during the 3 days preceding the illness onset date for cases, and the 3 days prior to the interview day for controls. The 3 days exposure experience encompasses the common incubation period for salmonellosis and has been used in other similar studies $[10,11]$. Parents or caretakers were given the option to fill out a self-administered mail-in questionnaire or participate in a 15 to 20 minute phone interview with a trained interviewer. The questionnaire was pilot-tested on volunteer parents, issues identified in this exercise were addressed, and appropriate changes were incorporated into the final version of the questionnaire. An informed consent was obtained from parents or caretaker prior to administering the questionnaire. The study protocol was reviewed and approved by the IRBs at Michigan State University and $\mathrm{MDCH}$.

\section{Data management and statistical analysis}

An age-matched ( $<1$ year, $2-<6$ years, and 6-10 years) pair univariable analysis of cases and controls using the conditional logistic regression was performed to examine associations between Salmonella infections and hypothesized risk factors. A multivariable analysis was conducted to identify factors associated with Salmonella infections, simultaneously controlling for potential confounders including race and household income, and matched odds ratio (MOR) and respective 95\% confidence interval (95\% $\mathrm{CI})$ was computed for each predictor variable. All variables with a p values $<0.25$ on univariable analyses, along with those hypothesized risk factors for Salmonella infections, were initially considered for inclusion in the multivariable model. After identifying the correlated variables, we examined the impact of collinearity by separately entering the variables (e.g., income, education, and race) into the multivariable regression model. We used the backward elimination method to obtain a parsimonious 
but yet plausible model. Additionally, to investigate potential effect modification, we also included several two way interaction terms in the model, 'household income and race,' 'household income and reptile ownership,' and 'and reptile ownership. A p-value $<0.05$ was considered statistically significant in all analysis. SAS was used to analyze the data.

Population attributable risk (PAR) for selected variables were estimated from the final multivariable model using Levin's formula for the calculation [12].

\section{Results}

A total of 123 cases representing a participation rate of $72.78 \%(123 / 169)$ were enrolled. A total of 139 control children were enrolled using one of the following two methods. Additional files 2 and 3 describe the enrollment of cases and controls respectively and list the number and type of exclusion criteria that were encountered during this study.

The majority (85\%) of the cases were interviewed within 20 days from the date when their records were available in the MDSS database. Control child was enrolled in parallel with cases enrollment however the cases were not individually matched with controls on the time of enrollment.

Comparison of the enrolled cases and controls is presented in Table 1. The enrolled cases and controls did not differ by socioeconomic and demographic characteristics including parental education, and annual household income except for distribution of racial composition $(p<$ $0.01)$.

Tables 2 and 3 show the results of univariable and multivariable logistic regression models respectively. The final multivariable model after adjusting for race, household income, and other known risk factors for salmonellosis [contact with a person having GI symptoms and other food related exposure (eating egg/meat/poultry) in the past 3 days] revealed that Salmonella infections were significantly associated with attending a daycare center $(\mathrm{MOR}=5.00(95 \% \mathrm{CI}: 1.51-16.58)$, contact with cats $(\mathrm{MOR}=2.53$ (95\% CI: 1.14-5.88), and contact with reptiles $(\mathrm{MOR}=7.90$ (95\% CI: $1.52-41.01)$. None of the twoway interactions tested (household income and race, household income and reptile ownership, and race and reptile ownership) were statistically significant ( $p>0.05)$. Reptile exposure had the highest PAR\% (35.61) among the risk factors identified for salmonellosis in our study.

\section{Discussion}

We found that cases of laboratory-confirmed salmonellosis were associated with several potentially modifiable risk factors and thus findings of this study can be used for enhancing salmonellosis prevention efforts in Michigan. Concurring with other investigations, our data suggest that children attendance of a daycare significantly increases the odds of Salmonella infections. Infections with several enteric agents have been associated with attending daycare $[8,12,13]$. The spread of infections in daycare centers is facilitated by crowding and microbial contamination of the environment [14]. Simple measures to control and prevent infections such as washing hands with soap and water after changing diapers, after assisting children with the toilet, and before handling food would help to substantially reduce the incidence of infections related to daycare.

We have found that contact with animals including reptile was associated with significant risk for salmonellosis in Michigan children. Salmonellosis is a well-recognized bacterial zoonoses [15]. Animals are the predominant reservoirs for the bacteria, and the prevalence of Salmonella carriage varies by species [16]. An estimated $90 \%$ of all reptiles including turtles and iguanas, carry and shed Salmonella in their feces intermittently [17]. Salmonella serotypes have been isolated from most vertebrates including dogs and cats with carriage rates of up to $36 \%$ and $18 \%$, respectively [18]. However, a much higher (up to $94 \%)$ Salmonella carriage rate has been observed in reptiles and amphibians [19]. In a recent case-control study conducted using FoodNet sites, reptile ownership was associated with more than 5-fold increased risk of salmonellosis in children aged $<1$ year [1]

In contrast to other similar studies [20] that reported an association between reptile-associated Salmonella specific serotypes (e.g., S. Stanley, S. Poona, S. Litchfield) and contact with reptile, our study did not find such association. This could be because of the limited sample size when the data were stratified by serotypes. We did not find significant differences in the frequency of Salmonella serotypes isolated from case children exposed to only reptiles or only cats. A recent study on Salmonella serotypes in captive reptiles reported a high diversity of Salmonella serotypes among reptile, which did not include serotypes Stanley, Poona or Litchfield [21]. Additionally, the MDCH registry-based study classified 59 isolates of Salmonella serotypes associated with children cases to be reptile-associated. The classical reptiles-associated Salmonella serotypes were not reported [22]. In this study, we found serotype Enteritidis and Typhimurium, which are frequent contaminants of chicken and meats, to be common among case children with reported contact with reptiles or cats. These findings are consistent with the previous report [22]. This assumed cycle of transmission may have resulted from foods contaminated with these serotypes that was fed to these animals [21].

Food-related exposures such as consumption of chicken, meat, and eggs/egg-containing product (established risk factors for salmonellosis in adults) did not show a significant association with Salmonella infections. 
Table 1: Socioeconomic characteristics of children aged $\leq 10$ years enrolled in a population-based case-control study to identify risk factors for Salmonella infections, Michigan, 2007.

\begin{tabular}{|c|c|c|c|c|}
\hline \multirow[t]{2}{*}{$\begin{array}{l}\text { Socioeconomic } \\
\text { characteristics }\end{array}$} & \multicolumn{2}{|l|}{$\begin{array}{l}\text { Cases } \\
n=123\end{array}$} & \multicolumn{2}{|l|}{$\begin{array}{l}\text { Controls } \\
n=139\end{array}$} \\
\hline & No. & (\%) & No. & $(\%)$ \\
\hline \multicolumn{5}{|l|}{ Age (year) } \\
\hline$<1$ & 22 & (17.89) & 25 & $(17.9$ \\
\hline $1-<6$ & 66 & $(53.66)$ & 66 & $(47.4$ \\
\hline $6-10$ & 35 & (28.46) & 48 & (34.5 \\
\hline
\end{tabular}

Sex

Female

Male

66

57

(53.66)

(46.34)

Race

Caucasians

African-Americans

Other minorities

(e.g., Asian, Alaskan

Indian)

\section{Parental education}

Elementary to High

school

Some college to

college degree

Post-graduate

degree

Refused to answer

Annual income

household

$<\$ 35,000$
$\$ 35,001-\$ 50,000$
$\$ 50,001-\$ 75,000$
$>\$ 75,000$
Refused to answer

(21.31)

(87.70)

(7.38)

(4.92)

6

(24.39)

$(58.54)$

(15.45)

(1.63)

(13.93)

(22.95)

(31.15)

(10.66)

13

Area of residence

High income:
$\$>60000$

Medium income:

$\$ 38000-\$ 60000$

Low income: $\$<38000^{\delta}$

17
(21.58)

${ }^{*}$ significant at $P<0.05$. P-value were computed using chi-square test

$\delta$ Categorized based on zip code level median household income obtained from the US Bureau of Census, 2000. 
Table 2: Univariable analyses of putative risk factors for Salmonella infections in children aged $\leq 10$ years, assessed in a population-based case- control study, Michigan. 2007.

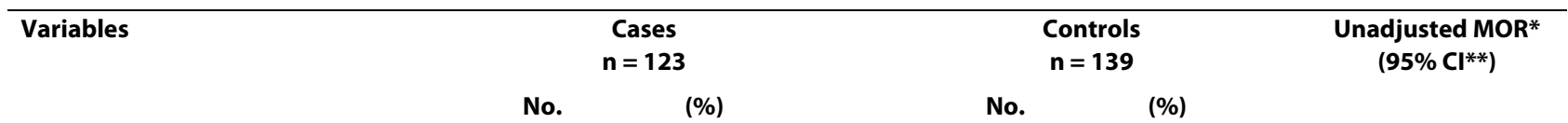

Household related variables

Number of people in household

$\begin{array}{lllllc}\leq 4 & 82 & (66.67) & 77 & (55.40) & \text { Reference } \\ >4 & 41 & (33.33) & 62 & (44.60) & 1.56(0.94-2.59)\end{array}$

Number of children aged $\leq 10$ years

$\begin{array}{lll}1 & 40 & (35.52) \\ 2-3 & 73 & (59.35) \\ \geq 3 & 10 & (8.13)\end{array}$

Number of bedrooms

$\begin{array}{lll}>3 & 36 & (29.27) \\ 2-3 & 70 & (56.91) \\ \leq 2 & 17 & (13.82)\end{array}$

Family room flooring

$\begin{array}{lll}\text { Wood } & 17 & (13.82) \\ \text { Carpet } & 88 & (71.54) \\ \text { Other and combination } & 18 & \text { (14.63) } \\ \text { end a daycare } & & \end{array}$

Attend a daycare

$\begin{array}{lcc}\text { No } & 106 & (86.18) \\ \text { Yes } & 17 & (13.82)\end{array}$

$(86.18)$
$(13.82)$

Child attends school other than daycare

$\begin{array}{llllll}\text { No } & 69 & (56.10) & 72 & (51.81) & \text { Reference } \\ \text { Yes } & 54 & (43.92) & 67 & (48.20) & 0.96(0.49-1.90)\end{array}$

\section{Food related exposures}

\section{Ate eggs/egg containing product}

\begin{tabular}{|c|c|c|c|c|c|}
\hline No & 44 & $(40.74)$ & 60 & $(44.44)$ & Reference \\
\hline Yes & 64 & $(59.26)$ & 75 & $(55.56)$ & $1.14(0.65-1.98)$ \\
\hline \multicolumn{6}{|l|}{ poultry } \\
\hline No & 13 & $(11.30)$ & 17 & $(12.50)$ & Reference \\
\hline Yes & 78 & $(67.83)$ & 95 & $(69.85)$ & $1.07(0.49-2.34)$ \\
\hline \multicolumn{6}{|l|}{ poultry at: } \\
\hline Home & 15 & $(41.67)$ & 78 & $(56.52)$ & Reference \\
\hline Outside home at a restaurant & 3 & $(8.33)$ & 3 & $(2.17)$ & $0.94(0.35-2.55)$ \\
\hline Both home and outside home & 4 & $(11.11)$ & 10 & $(7.25)$ & $3.37(0.86-13.21)$ \\
\hline \multicolumn{6}{|l|}{ meat } \\
\hline No & 36 & $(30.51)$ & 51 & $(37.78)$ & Reference \\
\hline Yes & 58 & $(49.15)$ & 60 & $(44.44)$ & $1.31(0.78-2.40)$ \\
\hline
\end{tabular}


Table 2: Univariable analyses of putative risk factors for Salmonella infections in children aged $\leq 10$ years, assessed in a population-based case- control study, Michigan. 2007. (Continued)

\begin{tabular}{|c|c|c|c|c|c|}
\hline \multicolumn{6}{|l|}{ Ate meat at: } \\
\hline Home & 9 & $(25.00)$ & 46 & (33.33) & Reference \\
\hline Outside home at a restaurant & 2 & $(5.56)$ & 6 & $(4.35)$ & $1.05(0.31-3.53)$ \\
\hline Both home and outside home & 4 & $(11.11)$ & 4 & $(2.90)$ & $1.36(0.32-5.20)$ \\
\hline \multirow[t]{2}{*}{ Univariable analysis } & Cases & & Controls & & $\begin{array}{l}\text { Un-adjusted MOR* } \\
\left(95 \% \mathrm{Cl}^{* *}\right)\end{array}$ \\
\hline & $n=123$ & (\%) & $n=139$ & (\%) & \\
\hline \multicolumn{6}{|l|}{ Drinking water source } \\
\hline Bottled & 25 & $(20.33)$ & 38 & 27.34 & Reference \\
\hline Municipal tap & 73 & $(59.35)$ & 81 & $(58.27)$ & $1.01(0.62-1.71)$ \\
\hline Private well water & 25 & $(20.33)$ & 20 & $(14.39)$ & $1.542(0.70-2.89)$ \\
\hline \multicolumn{6}{|l|}{ Family Kitchen practices } \\
\hline \multicolumn{6}{|l|}{ Keeps eggs refrigerated } \\
\hline Always & 118 & $(95.93)$ & 136 & $(97.84)$ & Reference \\
\hline Never or sometimes & 5 & $(4.07)$ & 3 & $(2.16)$ & $1.92(0.45-8.22)$ \\
\hline \multicolumn{6}{|l|}{ Clean kitchen counters with } \\
\hline Soap and disinfectant & 45 & $(36.89)$ & 70 & $(50.36)$ & Reference \\
\hline Soap and water only & 26 & $(21.31)$ & 25 & $(17.99)$ & $1.56(0.84-3.03)$ \\
\hline Disinfectant only & 51 & $(41.80)$ & 44 & $(31.65)$ & $1.75(1.01-3.04)$ \\
\hline \multicolumn{6}{|l|}{ How often clean kitchen counter } \\
\hline Daily & 109 & $(90.08)$ & 130 & (93.53) & Reference \\
\hline \multicolumn{6}{|l|}{ More than once a week/once a } \\
\hline Week/less than once a week & 12 & $(9.92)$ & 9 & $(6.47)$ & $1.51(0.61-3.73)$ \\
\hline
\end{tabular}

\section{Other environmental exposure}

\section{Handled packages of raw meat/} eggs while shopping with child

\begin{tabular}{|c|c|c|c|c|c|}
\hline $\begin{array}{l}\text { Did not go to shopping with } \\
\text { child }\end{array}$ & 64 & $(52.03)$ & 87 & (62.59) & Reference \\
\hline $\begin{array}{l}\text { Handled packages with } \\
\text { plastic/gloves }\end{array}$ & 15 & $(12.20)$ & 16 & $(11.51)$ & $1.26(0.54-2.76)$ \\
\hline $\begin{array}{l}\text { Handled packages without } \\
\text { plastic/gloves }\end{array}$ & 44 & $(35.77)$ & 36 & $(25.90)$ & $1.62(0.94-2.90)$ \\
\hline \multicolumn{6}{|l|}{$\begin{array}{l}\text { ntact with a person having GI } \\
\text { set }\end{array}$} \\
\hline No & 96 & $(78.05)$ & 123 & $(88.49)$ & Reference \\
\hline Yes & 27 & $(21.95)$ & 16 & $(11.51)$ & $2.15(1.10-4.21)$ \\
\hline \multicolumn{6}{|l|}{ ntact with animal } \\
\hline No contact with any animal & 42 & (34.15) & 81 & $(58.27)$ & Reference \\
\hline Any animal contact & 81 & $(65.85)$ & 58 & $(41.73)$ & $2.63(1.59-4.34)$ \\
\hline Dogs & 53 & (43.09) & 47 & (33.81) & $1.45(0.88-2.39)$ \\
\hline
\end{tabular}


Table 2: Univariable analyses of putative risk factors for Salmonella infections in children aged $\leq 10$ years, assessed in a population-based case- control study, Michigan. 2007. (Continued)

\begin{tabular}{lccccc}
\hline Cats & 35 & $(28.46)$ & 21 & $(15.11)$ & $2.23(1.22-4.11)$ \\
Reptiles & 17 & $(13.82)$ & 5 & $(3.60)$ & $4.16(1.49-11.62)$ \\
Birds & 4 & $(3.25)$ & 1 & $(0.72)$ & $4.57(0.50-41.33)$ \\
Hamster & 1 & $(0.81)$ & 2 & $(1.44)$ & $0.68(0.06-7.70)$
\end{tabular}

Travel history

\begin{tabular}{llllll}
\hline No & 90 & $(73.17)$ & 106 & $(76.26)$ & Reference \\
Yes & 31 & $(25.20)$ & 33 & $(23.74)$ & $1.08(0.61-1.91)$ \\
\hline
\end{tabular}

*Matched Odds ratio calculated using conditional logistic regression, ${ }^{* *}$ Confidence interval. All exposure data were gathered for during the 3 days of child's illness onset for cases and 3 days before the interview for controls.

It is conceivable the actual magnitude of food exposure related risk for Salmonella infections was not demonstrated in this study due to several factors. First, we obtained exposure information from interviewing surrogate sources (i.e., parents or caretakers). Secondly, certain exposures such as contact with animals are more likely to be accurately recalled, but the consumption of specific foods is more difficult to recall and thus prone to measurement error.

Exposure to contaminated environment was more commonly reported than exposure to contaminated food vehicles as a possible source for Salmonella infections in children $[7,23]$. Results of this study are in agreement with the findings of a case-control study conducted in the UK, where none of the household-related variables showed significant associations with Salmonella infection [24]. A recent study reported that children aged $<1$ year who ride in a shopping cart with meat or poultry placed next to them have a 4-fold increased risk for salmonellosis [1]. Although some studies [8,24] have reported the isolation of Salmonella from household dust, soil samples near the home, and samples from bathrooms, we have not microbiologically evaluated contamination of the household environment.

This was the first population-based case-control study in Michigan conducted to identify risk factors for salmo-

Table 3: Multivariable analysis of putative risk factors for Salmonella infections in children aged $\leq 10$ years, assessed in a population-based case-control study, Michigan, 2007.

$\begin{array}{lcc}\text { Variable } & \text { Unadjusted MORa } & \text { Adjusted MOR } \\ (95 \% \mathrm{Clb}) & \text { PARC } \\ & & (95 \% \mathrm{Clb})\end{array}$

\begin{tabular}{|c|c|c|c|}
\hline \multicolumn{4}{|c|}{ Contact with cats } \\
\hline No & Reference & Reference & \\
\hline Yes & $2.23(1.22-4.11)$ & $2.53(1.14-5.88)$ & $24.29(4.32-50.73)$ \\
\hline \multicolumn{4}{|c|}{ Contact with reptiles } \\
\hline No & Reference & Reference & \\
\hline Yes & $4.16(1.49-11.62)$ & $7.90(1.52-41.01)$ & $35.61(4.0-76.20)$ \\
\hline \multicolumn{4}{|c|}{ Attended a daycare } \\
\hline No & Reference & Reference & \\
\hline Yes & 2. $28(0.98-5.32)$ & $5.00(1.51-16.58)$ & $28.36(4.80-60.72)$ \\
\hline
\end{tabular}


nellosis in children. Although not all reported cases were enrolled in the study, participants and non-participants were drawn from the same base population, and the two groups were similar with respect to demographic characteristics $(\mathrm{p}>0.05)$. Our data were limited to laboratorydiagnosed cases only, and thus are biased (reporting bias) by factors that affect the probability of an illness being reported; caretakers of case children with mild to moderate disease symptoms may not seek medical care (selection bias) [5]. Further, recall bias may be present in the measurement of some exposure variables. Parents knew the disease status of their children prior to the interview and this may have influenced their responses [25]. We did not obtain specimens for culture from controls to exclude asymptomatic cases of salmonellosis. It is possible that some of our control children were asymptomatic Salmonella carriers and thus would have been misclassified. However, given the very low (1\%) prevalence of chronic carriers of Salmonella in healthy populations, we expect minimal misclassification due to this phenomenon [26].

\section{Conclusions}

This study revealed that attending a daycare center and contact with cats and reptiles within three days before a child's illness onset are important risk factors for infection with Salmonella serotypes in Michigan children. Data suggest that the contribution of environmental sources plays an important role in the acquisition of Salmonella infections in children. Further efforts are needed to educate parents and caretakers about the risk of Salmonella transmission to children from cats and reptiles, in addition to individuals having GI symptoms.

\section{Additional material}

Additional file 1 Study questionnaire. This questionnaire contains demographic, exposure including food intake information before the onset of the disease for cases and before the interview for control.

Additional file 2 Michigan Salmonella case-control study, 2007: Enrollment of cases (12/15/06 - 10/15/2007). All cases of children 10 years or younger in Michigan who were confirmed by laboratory methods to be a case of non-typhoidal Salmonella infection and signed a consent form, were enrolled.

Additional file 3 Michigan Salmonella case-control study, 2007: Enrollment of controls (12/15/06 - 10/15/2007). All non-case children who were matched on age and gender with cases and signed a consent form were enrolled.

Competing interests

The authors declare that they have no competing interests.

\section{Authors' contributions}

MY and AMS designed the study, conducted data analyses, and led the writing of the manuscript. MJW and HDD contributed to the study design and final data analyses. MHR, AAS and JF contributed to the data analysis and evaluation of results. CN participated in the data evaluation and coordination. SC and AMS oversaw the project, and helped in writing and the completion of the manuscript. All authors reviewed and approved the manuscript.

\section{Acknowledgements}

This research was supported in part by the NIH-Contract No. N01-Al-30058. (Microbiology Research Unit, MSU), while supplemental funds were provided by the Office of the Research Associate Dean of the College of Veterinary Medicine at Michigan State University. Additionally, M. Younus received a Food, Nutrition and Chronic Disease fellowship from the College of Human Medicine, MSU to conduct this study.

We sincerely thank our participants for their time and contribution. We thank Nicole Crisp, Diane Sinawi, Symone Coleman, Laya Keyvan, Amy Steffey, and Chiko Obi (members of Saeed's Lab at National Food Safety and Toxicology, MSU) for their efforts in data collection and managing study logistics at MSU, and Paula Jager and Nazneen Syed (Administrative Assistants, Communicable Disease Division, $\mathrm{MDCH}$ ) for providing study-related logistical support.

\section{Author Details}

1Department of Epidemiology, Michigan State University, East Lansing, Michigan 48824, USA, 2 National Food Safety and Toxicology, Michigan State University, East Lansing, Michigan 48824, USA, ${ }^{2}$ Large Animal Clinical Sciences, Michigan State University, East Lansing, Michigan 48824, USA, ${ }^{4}$ Communicable Disease Division, Michigan Department of Community Health, 201 Townsend Street, Lansing, Michigan 48913, USA, 5Departments of Pediatrics and Human Development, Michigan State University, East Lansing, Michigan 48824, USA, ${ }^{6}$ Division of Epidemiology, The University of Texas School of Public Health at Houston, Houston, Texas 77030, USA and 7East 235 42ndStreet, (MS:150-3-78), Safety and Risk Management, Pfizer Inc, New York, NY 10017

Received: 25 August 2009 Accepted: 16 April 2010

Published: 16 April 2010

\section{References}

1. Jones TF, Ingram LA, Fullerton KE, Marcus R, Anderson BJ, McCarthy PV, Vugia D, Shiferaw B, Haubert N, Wedel S, Angulo FJ: A case-control study of the epidemiology of sporadic Salmonella infection in infants. Pediatrics 2006, 118(6):2380-2387.

2. Voetsch AC, Van Gilder TJ, Angulo FJ, Farley MM, Shallow S, Marcus R, Cieslak PR, Deneen VC, Tauxe RV: FoodNet estimate of the burden of illness caused by nontyphoidal Salmonella infections in the United States. Clin Infect Dis 2004, 38(Suppl 3):S127-134.

3. CDC: Centers for Disease Control and Prevention. Preliminary FoodNet Data on the Incidence of Infection with Pathogens Transmitted Commonly Through Food - Selected Sites, United States, 2003. Morb Mortal Wkly Rep 2004, 53(16):338-343.

4. MDCH: Michigan Department of Community Health. Salmonellosis: Reportable Infectious Disease in Michigan (2002-2006). 2007 [https:// michigan.gov/documents/mdch/2005 CD Epi Profile 177917 7.pdf.

5. Younus M, Wilkins MJ, Arshad MM, Rahbar MH, Saeed AM: Demographic risk factors and incidence of Salmonella enteritidis infection in Michigan. Foodborne Pathog Dis 2006, 3(3):266-273.

6. Kimura AC, Reddy V, Marcus R, Cieslak PR, Mohle-Boetani JC, Kassenborg HD, Segler SD, Hardnett FP, Barrett T, Swerdlow DL: Chicken consumption is a newly identified risk factor for sporadic Salmonella enterica serotype Enteritidis infections in the United States: a casecontrol study in FoodNet sites. Clin Infect Dis 2004, 38(Suppl 3):\$244-252.

7. Delarocque-Astagneau E, Desenclos JC, Bouvet P, Grimont PA: Risk factors for the occurrence of sporadic Salmonella enterica serotype enteritidis infections in children in France: a national case-control study. Epidemiol Infect 1998, 121(3):561-567.

8. Haddock RL, Nocon FA: Infant salmonellosis and vacuum cleaners. Trop Pediatr 1994, 40(1):53-54.

9. $\mathrm{MDCH}$ : Health care professional's guide to the Michigan communicable disease rules. Lansing: Michigan Department of Community Health, 2001; 2001.

10. Cowden JM, O'Mahony M, Bartlett CL, Rana B, Smyth B, Lynch D, Tillett H, Ward L, Roberts D, Gilbert RJ, Baird-Parker AC, Kilsby DC: A national outbreak of Salmonella typhimurium DT 124 caused by contaminated salami sticks. Epidemiol Infect 1989, 103(2):219-225.

11. Sobel J, Hirshfeld AB, McTigue K, Burnett $C L$, Altekruse S, Brenner F Malcolm G, Mottice SL, Nichols CR, Swerdlow DL: The pandemic of Salmonella enteritidis phage type 4 reaches Utah: a complex 
investigation confirms the need for continuing rigorous control measures. Epidemiol Infect 2000, 125(1):1-8.

12. Levin ML: The occurrence of lung cancer in man. Acta Unio Int Contra Cancrum 1953, 9(3):531-541.

13. Goodman RA, Osterholm MT, Granoff DM, Pickering LK: Infectious diseases and child day care. Pediatrics 1984, 74(1):134-139.

14. Kotch JB, Weigle KA, Weber DJ, Clifford RM, Harms TO, Loda FA, Gallagher PN Jr, Edwards RW, LaBorde D, McMurray MP: Evaluation of an hygienic intervention in child day-care centers. Pediatrics 1994, 94(6):991-994.

15. Uzzau S, Brown DJ, Wallis T, Rubino S, Leori G, Bernard S, Casadesus J, Platt DJ, Olsen JE: Host adapted serotypes of Salmonella enterica. Epidemiol Infect 2000, 125(2):229-255.

16. Bender JB, Shulman SA: Reports of zoonotic disease outbreaks associated with animal exhibits and availability of recommendations for preventing zoonotic disease transmission from animals to people in such settings. J Am Vet Med Assoc 2004. 224(7):1105-1109.

17. Woodward DL, Khakhria R, Johnson WM: Human salmonellosis associated with exotic pets. J Clin Microbiol 1997, 35(11):2786-2790.

18. Annonymous: Selected zoonotic agents of gastroenteritis that can be acquired from dogs and cats: Salmonella. [http:// www.vetmed.wisc.edu/pbs/zoonoses/Glkgfel/salmonella.html]. Accessed on June 09, 2007

19. Mermin J, Hutwagner L, Vugia D, Shallow S, Daily P, Bender J, Koehler J, Marcus R, Angulo FJ: Reptiles, amphibians, and human Salmonella infection: a population-based, case-control study. Clin Infect Dis 2004 38(Suppl 3):S253-261

20. Warwick C, Lambiris AJ, Westwood D, Steedman C: Reptile-related salmonellosis. JR Soc Med 2001, 94(3):124-126

21. Pedersen K, Lassen-Nielsen AM, Nordentoft S, Hammer AS: Serovars of Salmonella from captive reptiles. Zoonoses Public Health 2009, 56(5):238-42.

22. Wells EV, Boulton M, Hall W, Bidol SA: Reptile-associated salmonellosis in preschool-aged children in Michigan, January 2001-June 2003. Clin Infect Dis 2004, 39(5):687-691.

23. Delarocque-Astagneau E, Bouillant C, Vaillant V, Bouvet P, Grimont PA, Desenclos JC: Risk factors for the occurrence of sporadic Salmonella enterica serotype typhimurium infections in children in France: a national case-control study. Clin Infect Dis 2000, 31(2):488-492.

24. Parry SM, Slader J, Humphrey T, Holmes B, Guildea Z, Palmer SR: A casecontrol study of domestic kitchen microbiology and sporadic Salmonella infection. Epidemiol Infect 2005, 133(5):829-835.

25. Schlesselman JJ, Stolley PD: Case-control Studies, Design, Conduct, Analysis. New York: Oxford University Press; 1982.

26. Devi S, Murray CJ: Salmonella carriage rate amongst school children-a three year study. Southeast Asian J Trop Med Public Health 1991, 22(3):357-361

doi: 10.1186/1756-0500-3-105

Cite this article as: Younus et al., Case-control study of disease determinants for non-typhoidal Salmonella infections among Michigan children BMC Research Notes 2010, 3:105

\section{Submit your next manuscript to BioMed Central} and take full advantage of:

- Convenient online submission

- Thorough peer review

- No space constraints or color figure charges

- Immediate publication on acceptance

- Inclusion in PubMed, CAS, Scopus and Google Scholar

- Research which is freely available for redistribution

Submit your manuscript at www.biomedcentral.com/submit
C Biomed Central 